1 Title: A Third Dose of SARS-CoV-2 Vaccine Increases Neutralizing Antibodies Against

2 Variants of Concern in Solid Organ Transplant Recipients

3 Authors: Andrew H. Karaba ${ }^{1}$, Xianming Zhu ${ }^{2}$, Tao Liang ${ }^{1}$, Kristy H. Wang ${ }^{1}$, Alex G.

4 Rittenhouse ${ }^{1}$, Olivia Akinde², Yolanda Eby², Jessica E. Ruff², Joel N. Blankson ${ }^{1}$, Aura T.

5 Abedon $^{3}$, Jennifer L. Alejo ${ }^{3}$, Andrea L. Cox ${ }^{1,4,5}$, Justin R. Bailey ${ }^{1}$, Elizabeth A.

6 Thompson ${ }^{1,5}$, Sabra L. Klein ${ }^{1,4}$, Daniel S. Warren ${ }^{3}$, Jacqueline M. Garonzik-Wang ${ }^{6}$,

7 Brian J. Boyarsky ${ }^{3}$, loannis Sitaras ${ }^{4}$, Andrew Pekosz ${ }^{1,4}$, Dorry L. Segev ${ }^{3}$, Aaron A.R.

8 Tobian $^{2^{*}}$, William A. Werbel ${ }^{1^{*}}$

\title{
9 Affiliations:
}

$10{ }^{1}$ Department of Medicine, Johns Hopkins University School of Medicine, Baltimore, MD 21287, USA.

$11{ }^{2}$ Department of Pathology, Johns Hopkins University School of Medicine, Baltimore, MD 21287, USA

$12{ }^{3}$ Department of Surgery, Johns Hopkins University School of Medicine, Baltimore, MD 21287, USA.

$13{ }^{4}$ W. Harry Feinstone Department of Molecular Microbiology and Immunology, Johns Hopkins University

14 Bloomberg School of Public Health, Baltimore, MD 21287, USA

$15{ }^{5}$ Bloomberg Kimmel Institute for Cancer Immunotherapy, Johns Hopkins University School of Medicine,

16 Baltimore, MD 21287, USA.

$17{ }^{6}$ Department of Surgery, University of Wisconsin School of Medicine and Health, Madison, WI 53792,

18 USA

19 *These authors contributed equally

22 Corresponding author email: wwerbel1@jhmi.edu 


\section{Abbreviations:}

32 Anti-N: Anti-nucleocapsid antibody

33 Anti-RBD: Anti-Receptor Binding Domain antibody

34 Anti-S: Anti-Spike antibody

35 AU: Arbitrary Unit

36 AUC: Area Under the Curve

37 CFR: Case Fatality Rate

38 CM: Complete Media

39 ELISA: enzyme-linked immunosorbent assay

40 HC: Healthy Control

41 IM: Infection Media

42 MSD: Meso Scale Diagnostics

43 nAb: Neutralizing antibody

44 NT50: 50\% Neutralization Titer

45 OD: Optical Density

46 SOTR: Solid Organ Transplant Recipient

47 TCID50: 50\% Tissue Culture Infectious Dose

48 VOC: Variant of Concern

49

50 Word Count: 2,898

51

52

53 


\section{ABSTRACT:}

55 Vaccine-induced SARS-CoV-2 antibody responses are attenuated in solid organ

56 transplant recipients (SOTRs) and breakthrough infections are more common.

57 Additional SARS-CoV-2 vaccine doses increase anti-spike IgG in some SOTRs, but it is

58 uncertain whether neutralization of variants of concern (VOCs) is enhanced. We tested

5947 SOTRs for clinical and research anti-spike IgG, pseudoneutralization (ACE2

60 blocking), and live-virus neutralization ( $\mathrm{nAb}$ ) against VOCs before and after a third

61 SARS-CoV-2 vaccine dose (70\% mRNA, 30\% Ad26.COV2.S) with comparison to 15

62 healthy controls after two mRNA vaccine doses. We used correlation analysis to

63 compare anti-spike IgG assays and focused on thresholds associated with neutralizing

64 activity. A third SARS-CoV-2 vaccine dose increased median anti-spike (1.6-fold) and

65 receptor-binding domain (1.5-fold) IgG, as well as pseudoneutralization against VOCs

66 (2.5-fold versus Delta). However, IgG and neutralization activity were significantly lower

67 than healthy controls $(p<0.001) ; 32 \%$ of SOTRs had zero detectable nAb against Delta

68 after third vaccination. Correlation with $n A b$ was seen at anti-spike $\lg G>4$ AU on the

69 clinical assay and $>10^{\wedge} 4 \mathrm{AU}$ on the research assay. These findings highlight benefits of

70 a third vaccine dose for some SOTRs and the need for alternative strategies to improve

71 protection in a significant subset of this population.

72

73

74

75

76 


\section{INTRODUCTION:}

Solid organ transplant recipients (SOTRs) are at increased risk for severe

COVID-19 ${ }^{1}$. For example, while the case fatality rate (CFR) for the general population in

80 the United States is $1-2 \%{ }^{2}$, CFRs range from $10-30 \%$ in SOTRs, due to a combination

81 of chronic disease and immunosuppressive medications ${ }^{3}$. Therefore, effective and

82 optimized vaccines that prevent COVID-19 disease in this group are critical.

83 Unfortunately, these patients were excluded from the phase III trials of the mRNA based

84 COVID-19 vaccines, ${ }^{4,5}$ and recent publications suggest that breakthrough disease is

85 more common among fully-vaccinated SOTRs than the general population ${ }^{6,7}$.

86 Furthermore, it has been demonstrated that many SOTRs develop weak SARS-CoV-2

87 antibody responses after the recommended two doses of an mRNA-based vaccine ${ }^{8-11}$.

88 This led to the hypothesis that a third dose of mRNA-based SARS-CoV-2 vaccine may

89 improve the immune response to SARS-CoV-2 and protection from COVID-19. Although

90 third doses have been authorized for immunocompromised persons in several

91 countries, including the United States (US), published data on neutralizing capacity of

92 SOTR plasma after additional vaccine doses are limited ${ }^{12-14}$. In particular, it is unknown

93 if augmented immune responses to a third vaccine dose would result in protection

94 against more transmissible variants of concern (VOCs) that exhibit immune escape,

95 including the Delta variant which currently comprises $>99 \%$ of new cases in the US ${ }^{15}$. In

96 an effort to assess whether a third dose of SARS-CoV-2 vaccine in SOTRs would

97 improve the SARS-CoV-2 specific neutralizing response, we measured total SARS-

98 CoV-2 specific IgG and neutralizing activity against the vaccine strain and four VOCs

99 before and after a third dose of SARS-CoV-2 vaccine and compared this to IgG levels 
100 and neutralizing capacity of healthy controls who received a standard two-dose mRNA-

101 based vaccine series.

102

\section{MATERIALS AND METHODS:}

2.1 Cohorts:

SOTR participants were enrolled in a national prospective, observational cohort: COVID-19 Antibody Testing of Recipients of Solid Organ Transplants and Patients with

107 Chronic Diseases, Johns Hopkins IRB00248540, as previously described ${ }^{9,16}$. Healthy 108 control participants were enrolled under Johns Hopkins IRB00027183 ${ }^{17}$. SOTRs

109 submitted blood samples to the investigators 0-4 weeks before and 2 weeks after third

110 vaccine doses, which were independently obtained in the community. Blood was

111 collected in Acid Citrate Dextrose tubes and plasma was isolated by Ficoll centrifugation

112 and stored at $-80^{\circ} \mathrm{C}$.

2.2 IgG Measurement:

Plasma was tested using the clinically available EUROIMMUN anti-SARS-CoV-2

$115 \lg$ enzyme-linked immunosorbent assay (ELISA) versus the S1 domain of spike

116 protein, performed per the manufacturers' protocols. Optical density (OD) of the sample

117 was divided by calibrator provided arbitrary unit $(\mathrm{AU})$ ratio, for which $\geq 1.1$ was

118 considered positive and $\geq 0.8-1.1$ were considered indeterminate. Plasma was thawed

119 and anti-N, anti-RBD, and anti-S IgG was measured using the multiplex

120 chemiluminescent Meso Scale Diagnostics (MSD) V-PLEX COVID-19 Respiratory

121 Panel 3 Kit according to the manufactures' protocol at a dilution of 1:5000. 
2.3 Pseudoneutralization/ACE2 Inhibition Measurement:

125 using the ACE2 MSD V-PLEX SARS-CoV-2 Panel 6 and Panel 14 kits according to the

126 manufacturers' protocol at a dilution of 1:100.

1272.4 Viruses and cells.

VeroE6-TMPRSS2 cells ${ }^{18}$ were cultured in complete media (CM) consisting of DMEM containing 10\% FBS (Gibco, Thermo Fisher Scientific), 1 mM glutamine

130 (Invitrogen, Thermo Fisher Scientific), $1 \mathrm{mM}$ sodium pyruvate (Invitrogen, Thermo

131 Fisher Scientific), $100 \mathrm{U} / \mathrm{mL}$ penicillin (Invitrogen, Thermo Fisher Scientific), and 100

$132 \mu \mathrm{g} / \mathrm{mL}$ streptomycin (Invitrogen, Thermo Fisher Scientific). Cells were incubated in a 5\%

$133 \mathrm{CO} 2$ humidified incubator at $37^{\circ} \mathrm{C}$.

The SARS-CoV-2/USA-WA1/2020 virus was obtained from BEI Resources. The

135 delta variant of SARS-CoV-2 (hCoV19/USA/MD-HP05660/2021, EPI_ISL_2331507)

136 was isolated on Vero-E6-TMPRSS2 cells plated in 24-well dishes and grown to $75 \%$

137 confluence. The CM was removed and replaced with $150 \mu \mathrm{L}$ of infection medium (IM),

138 which is identical to $\mathrm{CM}$ but with the fetal bovine serum reduced to $2.5 \%$, and $150 \mathrm{ul}$ of

139 the viral transport media containing a swab from a patient confirmed to be SARS-CoV-2

140 positive was added to the culture. The cultures were incubated at $37^{\circ} \mathrm{C}$ for 2 hours, the

141 inoculum was aspirated and replaced with $0.5 \mathrm{~mL}$ of IM and the cells cultured at $37^{\circ} \mathrm{C}$

142 for up to 5 days. When a cytopathic effect was visible in most of the cells, the IM was

143 harvested and stored at $-70^{\circ} \mathrm{C}$. The presence of SARS-CoV-2 was verified by

144 extracting RNA from the harvested supernatant using the Qiagen Viral RNA extraction

145 kit (Qiagen), and viral RNA detected using quantitative RT-PCR. ${ }^{19}$ The consensus 
146 sequence of the virus isolate did not differ from the sequence derived from the clinical

147 specimen.

The infectious virus titer was determined on VeroE6-TMPRSS2 cells using a

$14950 \%$ tissue culture infectious dose (TCID50) assay as previously described for SARS-

$150 \mathrm{CoV}^{20,21}$ Serial 10 -fold dilutions of the virus stock were made in IM, and then $100 \mu \mathrm{L}$ of

151 each dilution was added to the cells in a 96-well plate in sextuplicate. The cells were

152 incubated at $37^{\circ} \mathrm{C}$ for 4 days, visualized by staining with naphthol blue-black, and

153 scored visually for cytopathic effect.

1542.5 Neutralization assay.

The neutralizing antibody (nAb) levels were determined as described for SARS-

156 CoV. $^{22}$ Two-fold dilutions of plasma (starting at a 1:20 dilution) were made in IM.

157 Infectious virus was added to the plasma dilutions at a final concentration of $1 \times 104$

158 TCID50/mL (100 TCID50 per $100 \mu \mathrm{L})$. The samples were incubated for 1 hour at room

159 temperature, and then $100 \mu \mathrm{L}$ of each dilution was added to 1 well of a 96 -well plate of

160 VeroE6-TMPRSS2 cells in sextuplet for 6 hours at $37^{\circ} \mathrm{C}$. The inocula were removed,

161 fresh IM was added, and the plates were incubated at $37^{\circ} \mathrm{C}$ for 2 days or until complete

162 cytopathic effect was visible in wells exposed to virus without plasma. The cells were

163 fixed by the addition of $100 \mu \mathrm{L}$ of $4 \%$ formaldehyde per well, incubated for at least 4

164 hours at room temperature, and then stained with Napthol Blue Black (MilliporeSigma).

165 The nAb titer was calculated as the highest serum dilution that eliminated the cytopathic

166 effect in $50 \%$ of the wells and area under the curve (AUC) was calculated using

167 GraphPad Prism. 
2.6 Statistical analysis:

171 post third dose of SARS-CoV-2 vaccine were included in the analysis. Wilcoxon signed

172 rank test was used to compare the median of SARS-CoV-2 anti-Spike and anti-RBD

173 IgG level and percent ACE2 inhibition before and after third dose of vaccine among

174 SOTRs. The median of IgG level and ACE2 inhibition between SOTRs and HCs were 175 compared using Wilcoxon rank sum test. Pearson correlation was used to evaluate the

176 linear association between Spike IgG and percent ACE2 inhibition among SOTRs. A

177 spline knot was added at $4 \log _{10}(\mathrm{AU}) \mathrm{MSD}$ IgG. Bonferroni correction was conducted to

178 control multiple comparison when analyzing variants $(p<0.01$ was considered

179 statistically significant). The analysis was also stratified by type of third dose vaccine, 180 age, sex, and graft transplanted to evaluate effect measure modification. Missing values 181 were treated using available case strategy in subgroup analysis.

\section{RESULTS:}

3.1 Pre- and post-third dose samples were available for 47 SOTRs followed in our ongoing longitudinal observational cohort studying immunogenicity and safety of

186 SARS-CoV-2 vaccination. Most of these participants had previously undergone anti-

187 spike antibody testing using two clinically available assays ${ }^{16}$. The median age was 63

188 (interquartile range 49-70) years and 55\% were female. Most SOTRs were kidney

189 transplant recipients (64\%) and all initially received two doses of an mRNA-based

190 vaccine (23 Moderna mRNA-1273, 24 Pfizer BNT162b2). Most were taking a

191 calcineurin inhibitor-based maintenance immunosuppression regimen (77\%) and 30\% 
192 were on "triple immunosuppression" with a calcineurin inhibitor, an antimetabolite, and

193 corticosteroids. $70 \%$ of SOTRs received a third mRNA vaccine dose and $30 \%$ received

194 the Janssen Ad26.COV2.S vaccine. None reported a known history of COVID-19.

195 Among mRNA-vaccinated healthy controls ( $\mathrm{HC}, \mathrm{N}=15)$, none had known medical

196 conditions, and all received two doses of BNT162b2. See Table 1 for full demographic

197 and clinical data.

(N) total IgG were measured in plasma using a research assay (MSD) with FDA-verified sero-positivity cutoffs before and after a third dose of SARS-CoV-2 vaccine in SOTRs and after two doses of an mRNA-based vaccine in HCs. No participants had a positive 202 anti-N response at before or after a third dose of vaccine (Supplemental Figure 1). 203 Prior to a third dose of vaccine, 17 (36\%) and 11 (23\%) SOTRs were sero-positive for 204 anti-RBD and anti-S, respectively (Figure 1A). After the third dose, these numbers 205 increased to 36 (77\%) and 34 (72\%), respectively, and there was a significant increase

206 in the median total anti-S (1.6 fold change) and anti-RBD (1.5 fold change) lgG levels 207 compared to matched pre-third dose samples (Figure 1A). The median anti-RBD and 208 anti-S IgG values of SOTRs receiving a third dose remained significantly lower than the 209 median responses in fully vaccinated HCs after the two-dose series (Figure 1B). In 210 comparison to all other transplant recipients, kidney transplant recipients had

211 significantly lower anti-S IgG (Figure 1C). Eight (57\%) of those receiving Ad26.COV2.S 212 as a third dose and $26(79 \%)$ who received an mRNA-based vaccine as a third dose 213 became sero-positive. When stratifying by type of third dose received (mRNA versus 214 Ad.COV2.S), however, we did not observe a significant difference in median anti-S IgG 
215 value (Supplemental Figure 2C). In exploratory analysis, median IgG levels did not

216 differ by other key clinical or demographic parameters such as age or sex, though

217 subgroup sizes were small (Supplemental Figures 2A and 2B). Notably, seven female

218 kidney recipients had the lowest post third dose lgG levels of all SOTRs in the study. All

219 were taking anti-metabolite maintenance immunosuppression, but they did not

220 otherwise share clinical or demographic factors.

221 3.2 Next, we investigated the neutralizing potential of SOTR plasma versus major

222 SARS-CoV-2 VOCs after three vaccine doses with comparison to that of healthy

223 individuals after two vaccine doses. This was assessed initially via an assay measuring

224 capacity of plasma to inhibit S protein binding to the ACE2 receptor, termed

225 pseudoneutralization. There was a significant increase in the median

226 pseudoneutralization of all variants after a third vaccine dose among SOTRs: fold

227 changes 2.5, 2.2, 1.6, 1.5, and 2.5 for vaccine, Alpha, Beta, Gamma, Delta variants,

228 respectively (Figure 2A). However, pseudoneutralization of all variants was significantly

229 lower than that of healthy controls after two doses of an mRNA-based vaccine (Figure

230 2B). For example, only two (6\%) SOTRs had pseudoneutralization values for the Delta

231 variant above the first quartile of the healthy control pseudoneutralization values; the

232 majority were below $20 \%$ inhibition for all variants. When stratified by type of organ

233 received, kidney transplant recipients had significantly lower ACE2 inhibition versus the

234 vaccine strain and Alpha variant compared to all other organs (Figure 2C). Stratification

235 by age, sex, or vaccine platform, did not identify any significant differences in

236 pseudoneutralization (Supplemental Figure 3). 
We also examined the correlation between anti-S IgG and pseudoneutralization

238 for all the variants. We found a strong correlation between anti-S IgG and

239 pseudoneutralization, but the relationship only became linear around $4 \log _{10}($ arbitrary

240 unit, $A U) \lg$, suggesting that values below this may not correlate with neutralizing

241 response (Figure 2D).

3.3 Finally, we used live-virus neutralization (nAb) to assess 50\% neutralization

243 titer (NT50) and area under the curve (AUC) against the vaccine strain and the Delta

244 variant before and after a third vaccine dose in SOTRs and in two-dose vaccinated

245 HCs. For SOTRs, median (IQR) NT50s were 40 (10-120) versus vaccine strain and 20

246 (10-40) versus Delta (Figure 3A), with median (IQR) AUC of 50 (2-145) and 9 (3-50),

247 respectively (Figure $3 B$ ) after a third vaccine dose. This corresponded to a fold change

248 in NT50 of 1.6 and 1.3 and a fold change in AUC 50.2 and 8.4 versus the vaccine strain

249 and Delta variant, respectively. Compared to HCs, NT50s and AUC versus vaccine and

250 Delta variant strains were significantly lower among SOTRs (Figure 3D). Fully 32\% of

251 SOTRs had nAb NT50s at or below the limit of detection versus the Delta variant after a

252 third vaccine dose (as compared to $0 \%$ of $\mathrm{HCs}$ ). There were two female liver transplant

253 recipients with very high neutralizing titers, even beyond those of the HCs.

3.4 We assessed inter-assay correlation for both the vaccine strain (Figure 4A)

255 and Delta variants (Figure 4B) among clinical (EUROIMMUN) and research (MSD) anti-

256 spike IgG assays, as well as pseudoneutralization and nAb AUC for SOTRs and HCs.

257 For the vaccine strain, EUROIMMUN and MSD IgG showed excellent positive

258 correlation, particularly above the clinical manufacturer threshold for seropositivity (1.1

$259 \mathrm{AU})$. Correlation of pseudoneutralization with both $\lg \mathrm{G}$ assays was strong above a 
260 threshold of $20 \%$ ACE2 blocking. Below this, there was marked variation in

261 corresponding IgG levels among SOTRs (e.g., EUROIMMUN IgG ranged 0-7.5 AU)

262 (Supplemental Figure 4). Correlation of both IgG assays and nAb AUC was moderate,

263 though markedly improved when restricting to higher IgG cutoffs (4 AU on the

264 EUROIMMUN assay and $4 \log 10(\mathrm{AU})$ on the MSD assay). Overall correlation of

265 pseudoneutralization and nAb was stronger, particularly when restricting each to one

266 patient group (SOTR or HC). These cross-correlation patterns were similar when

267 considering the Delta variant, although pseudoneutralization and nAb AUC correlation

268 was overall stronger as compared to the vaccine strain, reflecting reduction in ACE2

269 blocking for HCs.

\section{DISCUSSION}

Here, we provide evidence that a third dose of COVID-19 vaccine increases

273 plasma neutralization against VOCs for some SOTRs, including versus the highly

274 transmissible and now dominant Delta variant. This was robustly characterized using a

275 combination of clinical and research IgG assays, pseudoneutralization, and gold-

276 standard live-virus neutralization. Although median plasma neutralizing capacity did

277 increase for SOTRs, levels were generally far below that of HCs after the two-dose

278 mRNA series and 32\% showed no nAb against the Delta variant using the live-virus

279 assay.

280 Other key findings include lower neutralization among kidney transplant

281 recipients versus other transplant recipients, potentially reflecting heavier maintenance

282 immunosuppression. Other factors previously associated with improved sero-response 
283 such as younger age or third dose vaccine platform (i.e., mRNA) were not clearly

284 associated with response. Importantly, although there was significant variability in $\lg G$

285 responses in SOTRs, we found evidence through correlation analysis that certain $\lg \mathrm{G}$

286 cutoffs were associated with clear increases in ACE2 blocking, as well as in nAb. This is

287 an early step toward establishing thresholds for high-throughput assays that may

288 indicate protection from COVID-19, including the Delta variant, though this will need to

289 be tested by assessing risk of infection in real-world cohort and clinical trial settings.

The observed variable humoral response to additional vaccine doses in this high-

291 risk group indicates that alternative strategies, such as immunosuppressive modulation

292 or using emerging vaccine platforms, may be necessary to induce a protective response

293 to vaccination. While some SOTRs clearly produce an antibody response on par with

294 HCs, they were a small minority in this study. Though we identified kidney

295 transplantation as a risk factor for decreased responsiveness, the mechanism

296 underlying this association is unknown. This is evident in the seven female kidney

297 recipients who all had very low IgG and neutralizing responses, yet had no clear pattern

298 in demographic features, age, or vaccine platform received as a third dose. Additional

299 investigations and deeper immunological analyses are warranted to understand in a

300 personalized fashion why some SOTRs respond to additional antigen exposure, while

301 others do not.

This study was limited by its observational nature and small number of

303 participants with demographic and immunosuppressive heterogeneity. Additionally, HC

304 comparators were younger than the SOTR group, which may contribute to observed

305 differences in humoral response. Although patient survey and anti-N IgG were used to 
306 rule out prior COVID-19, it is possible that subclinical infections occurred in some

307 patients before or after vaccination. Furthermore, mucosal immune responses and

308 cellular immune responses were not characterized in this study.

309 In summary, a third dose of a SARS-CoV-2 vaccine increases anti-spike and

310 anti-RBD IgG levels and plasma neutralizing capability, including against the Delta

311 variant, in some SOTRs. Yet, a significant portion of SOTRs have limited or no

312 neutralizing activity against the dominant $\mathrm{VOC}$ indicating that a third dose of vaccine

313 may not be a fully effective strategy for a large portion of immunocompromised patients.

314 These data also inform how research and clinical anti-spike IgG measurements might

315 be used to estimate neutralizing ability and potential sero-protection thresholds. This is

316 novel and timely information regarding the potential improvement of immune protection

317 against SARS-CoV-2 variants in a highly vulnerable population amidst ongoing

318 community surges.

\section{ACKNOWLEDGEMENTS:}

322 This work was supported by the Ben-Dov family, the Johns Hopkins COVID-19

323 Vaccine-related Research Fund, the National Cancer Institute (U54CA260491), grants

324 T32DK007713 (JLA), F32DK124941 (BJB), and K23DK115908 (JMGW) from the

325 National Institute of Diabetes and Digestive and Kidney Diseases, and grants

326 K24Al144954 (DLS), K08Al156021 (AHK), K23Al157893 (WAW),

327 HHSN272201400007C (AP) and R01Al120938S1 (AART) from the National Institute of

328 Allergy and Infectious Disease. 
AUTHOR CONTRIBUTIONS:

331 AHK and WAW conceived of the study and design. OA, JER, and YE processed the

332 samples and prepared them for the assays. AHK, KHW, and AGR performed the MSD

333 assays and collected the antibody data. AP and IS performed the live virus

334 neutralization. ATA, JLA, JNB, DW, and BJB assisted with participant enrollment and

335 collection of clinical data. XZ and TL performed the analysis. EAT assisted with sample

336 curation and data interpretation. AHK and WAW wrote the original manuscript. JMG,

337 ALC, JNB, SLK, AP, JRB, DLS, AART, and WAW supervised the studies, provided

338 material support, and contributed to the interpretation of results. All authors aided in

339 editing the manuscript.

341 DISCLOSURES:

342 DLS has the following financial disclosures: consulting and speaking honoraria from

343 Sanofi, Novartis, CSL Behring, Jazz Pharmaceuticals, Veloxis, Mallinckrodt, Thermo

344 Fisher Scientific. None of the other authors have any relevant competing interests.

DATA AVAILABILITY:

347 Reasonable requests for deidentified data to the corresponding author will be granted. 


\section{FIGURE LEGENDS}

\section{Figure 1. Changes in SARS-CoV-2 Specific IgG After a Third Dose of SARS-CoV-2}

\section{Vaccine}

A. Total SARS-CoV-2 S1 RBD (left) and Spike (right) specific IgG in SOTRs before and after a third dose of vaccine. The dashed line represents the assay manufacturer's cut-off for seropositivity based on convalescent samples.

B. Total SARS-CoV-2 S1 RBD (left) and Spike (right) specific IgG in fully mRNA vaccinated healthy controls $(H C s)(n=15)$ and SOTRs after a third dose of vaccine $(n=47)$. and non-kidney $(n=17)$ transplant.

The boxplots represent the IQR. The median is represented by a horizontal line in the box. The lower and upper whiskers represent 1.5x the IQR beyond the quartiles. Each dot represents an individual sample. Statistical differences between groups were determined by Wilcoxon signed rank test for panel A, and Wilcoxon rank sum test for panel B and C. P-values of $<0.05$ were considered significant. 
371 Figure 2. SARS-CoV-2 Pseudoneutralization After a Third Dose of COVID-19

372 Vaccine in SOTRs

373

374 A. Pseudoneutralization of full-length SARS-CoV-2 Spike variants (indicated in top 375 header of each panel) before and after a third dose of vaccine among SOTRs.

376 B. Pseudoneutralization of full-length SARS-CoV-2 Spike variants (indicated in top 377 header of each panel) in SOTRs $(n=47)$ after a third dose of vaccine compared 378 to fully vaccinated healthy controls $(n=15)$.

379 C. Comparison of pseudoneutralization of full-length SARS-CoV-2 Spike variants (indicated in top header of each panel) in SOTRs who received kidney $(n=30)$ and non-kidney $(\mathrm{n}=17)$ transplant.

D. Correlation between total SARS-CoV-2 Spike IgG and pseudoneutralization of full-length SARS-CoV-2 Spike variants among SOTRs receiving a third dose of vaccine.

387 beyond the quartiles. Each dot represents an individual sample. Statistical

388 differences between groups were determined by Wilcoxon signed rank test for panel

389 A, and Wilcoxon rank sum test for panel B and C. Pearson correlation coefficient 390 were generated for panel D. P-values of $<0.01$ were considered significant after $391 \quad$ Bonferroni correction. 

Delta variant

A. nAb NT50 versus SARS-CoV-2 vaccine strain and Delta variant before and after a third dose SARS-CoV-2 vaccine among SOTRs.

B. nAb area under curve (AUC) versus SARS-CoV-2 vaccine strain and Delta variant before and after a third dose SARS-CoV-2 vaccine among SOTRs. HCs after two mRNA vaccine doses between SOTRs after a third dose of SARS-CoV-2 vaccine and HCs after two mRNA vaccine doses. horizontal line in the box. The lower and upper whiskers represent 1.5x the IQR beyond the quartiles. Each dot represents an individual sample. Statistical

410 differences between groups were determined by Wilcoxon signed rank test for panel $411 \quad A$ and $B$, and Wilcoxon rank sum test for panel $C$ and $D$. 
416 Figure 4. Correlations between neutralizing antibody (nAb), percent ACE2 417 inhibition, MSD anti-spike IgG and EUROIMMUN anti-spike IgG of SARS-CoV-2 418 among SOTRs and HCs

A. Correlations between neutralizing and IgG assays versus the SARS-CoV-2 vaccine strain among SOTRs after a third dose of vaccine and HCs after two

422 doses.

423 B. Correlations between neutralizing and IgG assays versus the Delta variant among SOTRs after a third dose of vaccine and HCs after two doses.

425 Each point on the scatter plots represents an individual sample. Pearson correlation

426 coefficients between assays are presented in the upper panels. "Corr" represents

427 the correlation across all samples. "HCs" (in red) represents the correlation among

428 only HCs. "SOTR" (in blue) represents the correlation among only SOTRs. * $p<$

$4290.05 ;{ }^{* *} p<0.01 ;{ }^{* *} p<0.001$. Density plots of SOTRs and HCs are shown in

430 diagonal panels. Unit of analysis: nAb AUC, $\log _{10} A U C ; A C E 2:$ percent ACE2 


\section{Table 1. Clinical and Demographic Characteristics of SOTRs and Healthy}

\section{Controls.}

\begin{tabular}{|c|c|c|c|}
\hline & $\begin{array}{c}\text { Overall } \\
\mathrm{n}=62\end{array}$ & $\begin{array}{l}\text { SOTR } \\
n=47\end{array}$ & $\begin{array}{l}\text { Healthy controls, } \\
\qquad \mathrm{n}=15\end{array}$ \\
\hline \multicolumn{4}{|l|}{ Age, years } \\
\hline $20-39$ & $10(16)$ & $3(6)$ & $7(47)$ \\
\hline $40-59$ & $26(42)$ & $18(38)$ & $8(53)$ \\
\hline $60-79$ & $26(42)$ & $26(55)$ & $0(0)$ \\
\hline \multicolumn{4}{|l|}{ Sex } \\
\hline Female & $31(50)$ & $26(55)$ & $5(33)$ \\
\hline Male & $31(50)$ & $21(45)$ & $10(67)$ \\
\hline \multicolumn{4}{|l|}{ Race } \\
\hline White & $57(92)$ & $46(98)$ & $11(73)$ \\
\hline Asian & $4(6)$ & $1(2)$ & $3(20)$ \\
\hline African American & $1(2)$ & $0(0)$ & $1(7)$ \\
\hline \multicolumn{4}{|l|}{ Graft transplanted } \\
\hline Kidney* & - & $30(64)$ & - \\
\hline Liver & - & $10(21)$ & - \\
\hline Heart & - & $4(9)$ & - \\
\hline Lung & - & $2(4)$ & - \\
\hline Pancreas & - & $1(2)$ & - \\
\hline \multicolumn{4}{|l|}{ Anti-rejection medication $\dagger$} \\
\hline Prednisone & - & $22(47)$ & - \\
\hline Calcineurin Inhibitors & - & $36(77)$ & - \\
\hline mTOR inhibitors & - & $7(15)$ & - \\
\hline anti-metabolites & - & $30(64)$ & - \\
\hline \multicolumn{4}{|l|}{ Type of the third dose vaccine } \\
\hline mRNA & - & $33(70) \ddagger$ & - \\
\hline Ad26.COV2.S & - & $14(30)$ & - \\
\hline Days between second dose and third dose vaccine & - & $102(70-124)$ & - \\
\hline Days between transplant and third dose vaccine & - & $1778(930-4419)$ & - \\
\hline Days post second dose vaccine & - & - & $8(7-10)$ \\
\hline
\end{tabular}

Note: all study participants received mRNA vaccine for the first two doses. Categorical variables were presented in $\mathrm{n}(\%)$, and continuous variables were presented in median (interquartile range). *1 person had both kidney and 


\section{Supplemental Figure 1.}

448 Total SARS-CoV-2 Nucleocapsid specific IgG in SOTRs before and after a third dose of

449 vaccine. The dashed line represents the assay manufacturer's cut-off for positivity

450 based on convalescent samples. P-values were calculated using Wilcoxon rank sum

451 test.

Total SARS-CoV-2 Spike specific IgG in SOTRs who received a third dose of COVID-19

456 vaccine stratified by age $(<60 n=21$ and $\geq 60$ years $n=26)$, sex (female $n=26$ and

457 male $n=21$ ), and type of third dose vaccine received (mRNA $n=33$ and Ad26.COV2.S

$458 \mathrm{n}=14)$. P-values were calculated using Wilcoxon rank sum test and should be

459 considered exploratory given the small subgroups.

460

\section{Supplemental Figure 3.}

462 Pseudoneutralization of full-length SARS-CoV-2 Spike variants in SOTRs who received

463 a third dose of COVID-19 vaccine stratified by age $(<60 n=21$ and $\geq 60$ years $n=26)$,

464 sex (Female $n=26$ and male $n=21)$, and type of third dose vaccine received (mRNA $n$

$465=33$ and Ad26.COV2.S $n=14)$. P-values were calculated using Wilcoxon rank sum test

466 and should be considered exploratory given the small subgroups. 
470 Supplemental Figure 4. Correlation between percent ACE2 inhibition and

471 neutralizing antibody ( $\mathrm{nAb}$ ) and IgG of SARS-CoV-2 among SOTRs after a third

472 dose of COVID-19 vaccine, stratified by ACE2 inhibition level

473 A. Pearson correlation between vaccine strain percent ACE2 inhibition and vaccine

$474 \quad$ strain neutralizing antibody (nAb), MSD IgG and EUROIMMUN IgG of SARS-

475 CoV-2 among SOTRs after a third dose of COVID-19 vaccine.

476 B. Pearson correlation between Delta variant percent ACE2 inhibition and Delta

477 variant neutralizing antibody (nAb), MSD IgG and EUROIMMUN IgG of SARS-

478 CoV-2 among SOTRs after a third dose of COVID-19 vaccine.

479 Units of analysis: nAb AUC, $\log _{10} A \cup C$; ACE2: percent ACE2 inhibition; MSD IgG, $480 \quad \log _{10} \lg \mathrm{G} A U / \mathrm{mL} ;$ EUROIMMUN IgG, $\mathrm{AU} / \mathrm{mL}$.

481

482 
medRxiv preprint doi: https://doi.org/10.1101/2021.08.11.21261914; this version posted October 13, 2021. The copyright holder for this preprint (which was not certified by peer review) is the author/funder, who has granted medRxiv a license to display the preprint in perpetuity. It is made available under a CC-BY-NC-ND 4.0 International license .

\section{REFERENCES}

484 1. Fung M, Babik JM. COVID-19 in Immunocompromised Hosts: What We Know So Far. Clinical Infectious Diseases. 2021;72(2):340-350. doi:10.1093/cid/ciaa863

2. COVID-19 Map. Johns Hopkins Coronavirus Resource Center. Accessed June 15, 2020. https://coronavirus.jhu.edu/map.html

3. Raja MA, Mendoza MA, Villavicencio A, et al. COVID-19 in solid organ transplant recipients: A systematic review and meta-analysis of current literature. Transplantation Reviews. 2021;35(1):100588. doi:10.1016/j.trre.2020.100588

4. Baden LR, El Sahly HM, Essink B, et al. Efficacy and Safety of the mRNA-1273 SARS-CoV-2 Vaccine. New England Journal of Medicine. 2021;384(5):403-416. doi:10.1056/NEJMoa2035389

5. Polack FP, Thomas SJ, Kitchin N, et al. Safety and Efficacy of the BNT162b2 mRNA Covid-19 Vaccine. New England Journal of Medicine. 2020;383(27):26032615. doi:10.1056/NEJMoa2034577

6. Aslam S, Adler E, Mekeel K, Little SJ. Clinical effectiveness of COVID-19 vaccination in solid organ transplant recipients. Transplant Infectious Disease. $2021 ; n / a(n / a): e 13705$. doi:10.1111/tid.13705

7. Qin CX, Moore LW, Anjan S, et al. Risk of Breakthrough SARS-CoV-2 Infections in Adult Transplant Recipients. Transplantation. Published online July 23, 2021. doi:10.1097/TP.0000000000003907

8. Boyarsky BJ, Werbel WA, Avery RK, et al. Immunogenicity of a Single Dose of SARS-CoV-2 Messenger RNA Vaccine in Solid Organ Transplant Recipients. JAMA. 2021;325(17):1784-1786. doi:10.1001/jama.2021.4385

9. Boyarsky BJ, Werbel WA, Avery RK, et al. Antibody Response to 2-Dose SARSCoV-2 mRNA Vaccine Series in Solid Organ Transplant Recipients. JAMA. 2021;325(21):2204-2206. doi:10.1001/jama.2021.7489

10. Hall VG, Ferreira VH, lerullo $M$, et al. Humoral and cellular immune response and safety of two-dose SARS-CoV-2 mRNA-1273 vaccine in solid organ transplant recipients. American Journal of Transplantation. 2021;n/a(n/a). doi:10.1111/ajt.16766

11. Sattler A, Schrezenmeier E, Weber UA, et al. Impaired humoral and cellular immunity after SARS-CoV2 BNT162b2 (Tozinameran) prime-boost vaccination in kidney transplant recipients. J Clin Invest. Published online June 8, 2021. doi:10.1172/JCl150175 
medRxiv preprint doi: https://doi.org/10.1101/2021.08.11.21261914; this version posted October 13, 2021. The copyright holder for this preprint (which was not certified by peer review) is the author/funder, who has granted medRxiv a license to display the preprint in perpetuity. It is made available under a CC-BY-NC-ND 4.0 International license .

12. Hall VG, Ferreira VH, Ku T, et al. Randomized Trial of a Third Dose of mRNA-1273 Vaccine in Transplant Recipients. N Engl J Med. Published online August 11, 2021. doi:10.1056/NEJMc2111462

13. Kamar N, Abravanel F, Marion O, Couat C, Izopet J, Del Bello A. Three Doses of an mRNA Covid-19 Vaccine in Solid-Organ Transplant Recipients. New England Journal of Medicine. 2021;0(0):null. doi:10.1056/NEJMc2108861

14. Benotmane I, Gautier G, Perrin P, et al. Antibody Response After a Third Dose of the mRNA-1273 SARS-CoV-2 Vaccine in Kidney Transplant Recipients With Minimal Serologic Response to 2 Doses. JAMA. 2021;326(11):1063. doi:10.1001/jama.2021.12339

15. CDC. COVID Data Tracker. Centers for Disease Control and Prevention. Published March 28, 2020. Accessed August 6, 2021. https://covid.cdc.gov/covid-data-tracker

16. Werbel WA, Boyarsky BJ, Ou MT, et al. Safety and Immunogenicity of a Third Dose of SARS-CoV-2 Vaccine in Solid Organ Transplant Recipients: A Case Series. Ann Intern Med. Published online June 15, 2021. doi:10.7326/L21-0282

17. Woldemeskel BA, Karaba AH, Garliss CC, et al. The BNT162b2 mRNA Vaccine Elicits Robust Humoral and Cellular Immune Responses in People Living with HIV. Clinical Infectious Diseases. 2021;(ciab648). doi:10.1093/cid/ciab648

18. Matsuyama S, Nao N, Shirato K, et al. Enhanced isolation of SARS-CoV-2 by TMPRSS2-expressing cells. Proc Natl Acad Sci USA. 2020;117(13):7001. doi:10.1073/pnas.2002589117

19. Waggoner JJ, Stittleburg V, Pond R, et al. Triplex Real-Time RT-PCR for Severe Acute Respiratory Syndrome Coronavirus 2. Emerg Infect Dis. 2020;26(7):16331635. doi:10.3201/eid2607.201285

20. Schaecher SR, Touchette E, Schriewer J, Buller RM, Pekosz A. Severe Acute Respiratory Syndrome Coronavirus Gene 7 Products Contribute to Virus-Induced Apoptosis. Journal of Virology. 2007;81(20):11054-11068. doi:10.1128/JVI.0126607

21. Schaecher SR, Mackenzie JM, Pekosz A. The ORF7b Protein of Severe Acute Respiratory Syndrome Coronavirus (SARS-CoV) Is Expressed in Virus-Infected Cells and Incorporated into SARS-CoV Particles. Journal of Virology. 2007;81(2):718-731. doi:10.1128/JVI.01691-06

22. Schaecher SR, Stabenow J, Oberle C, et al. An immunosuppressed Syrian golden hamster model for SARS-CoV infection. Virology. 2008;380(2):312-321. doi:10.1016/j.virol.2008.07.026 

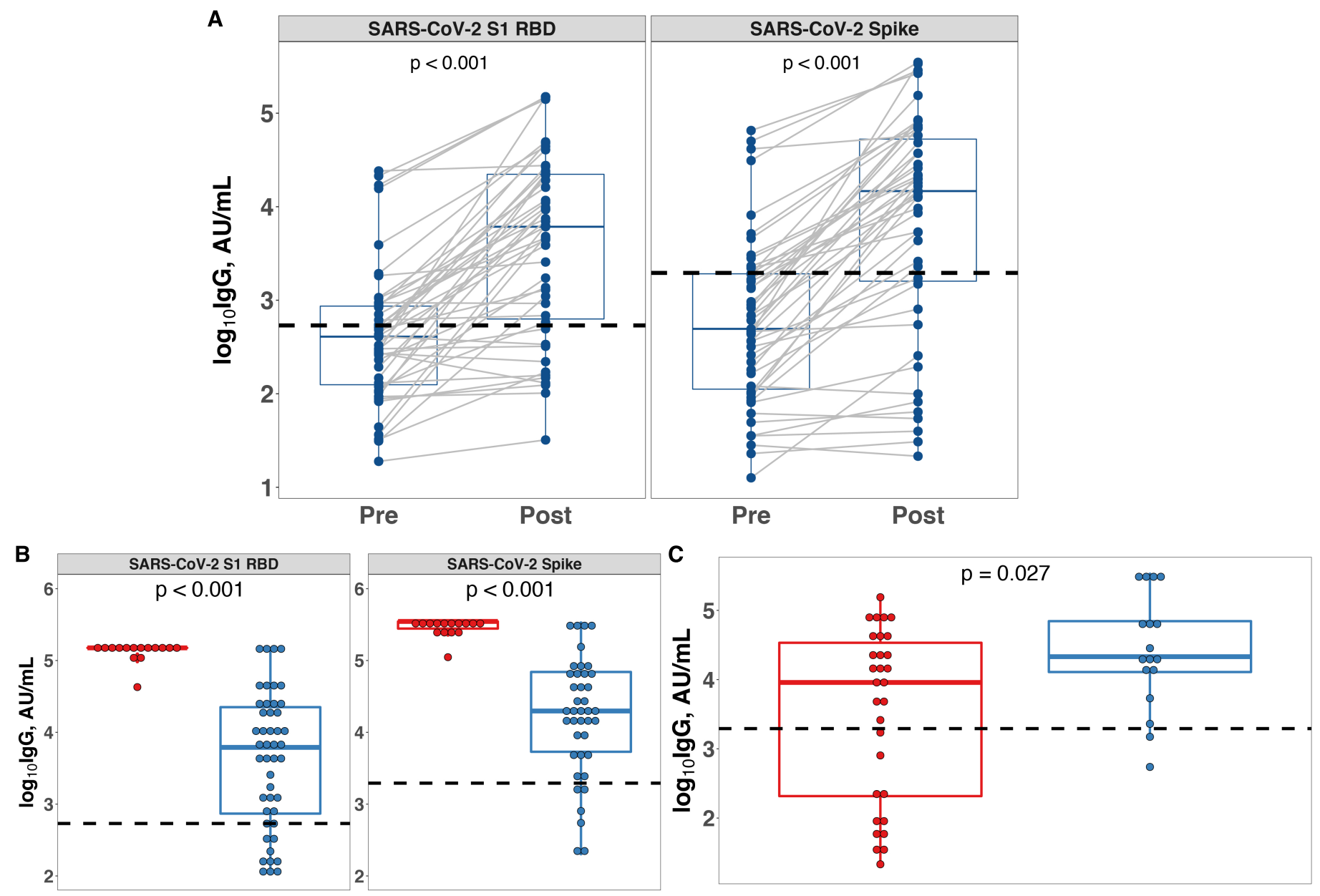

Cohort $\bullet$ HCs $\odot$ SOTRs

Organ Transplanted $\bullet$ Kidney $\bullet$ Other 
A

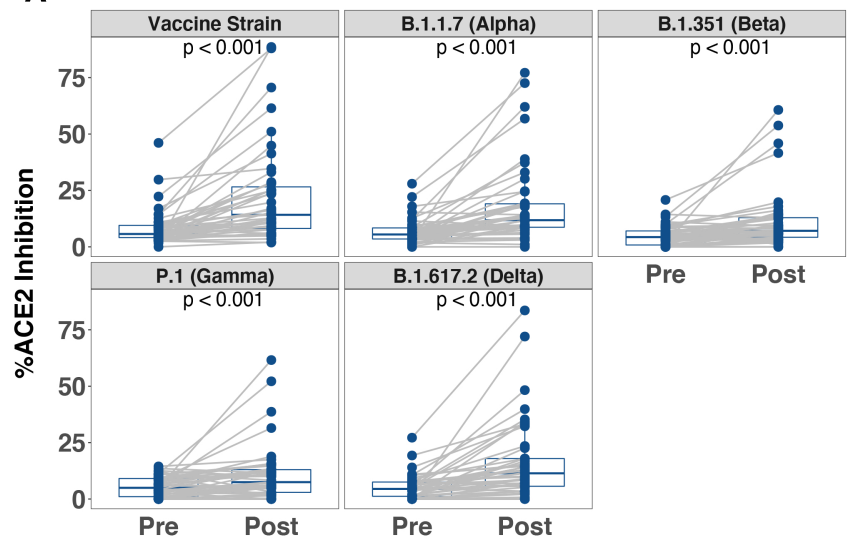

C

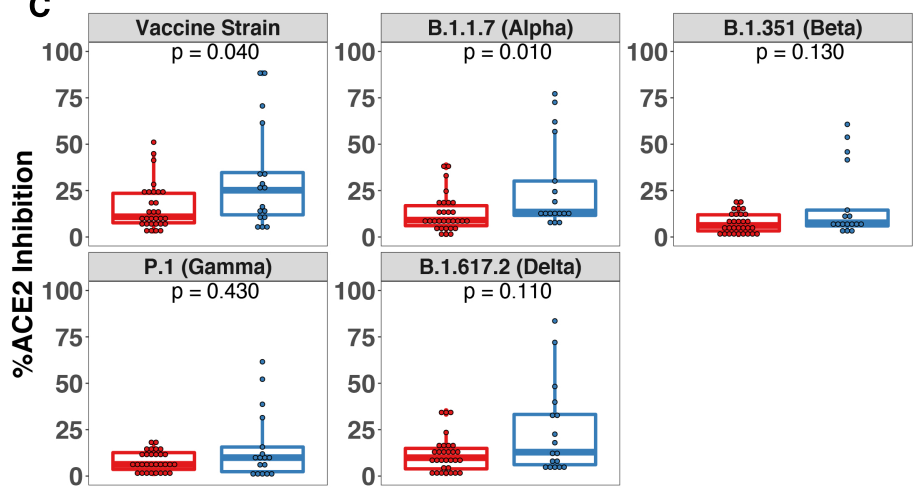

B
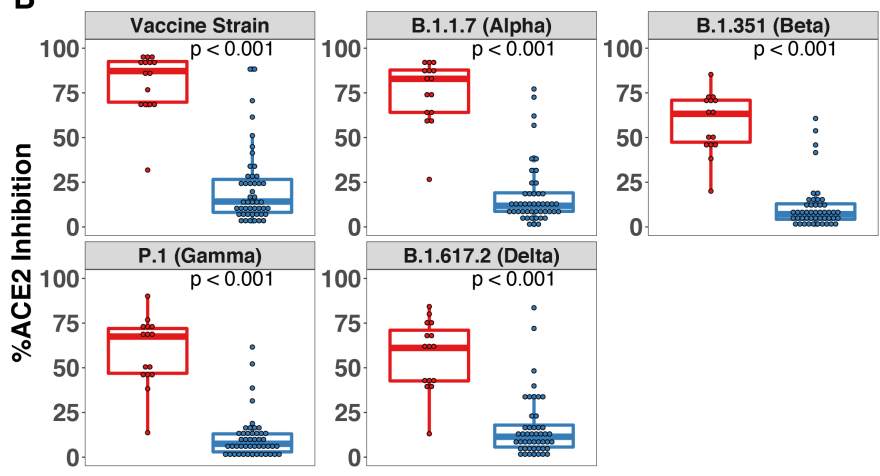

Cohort $\bullet$ HCs $\bigcirc$ SOTRs

D

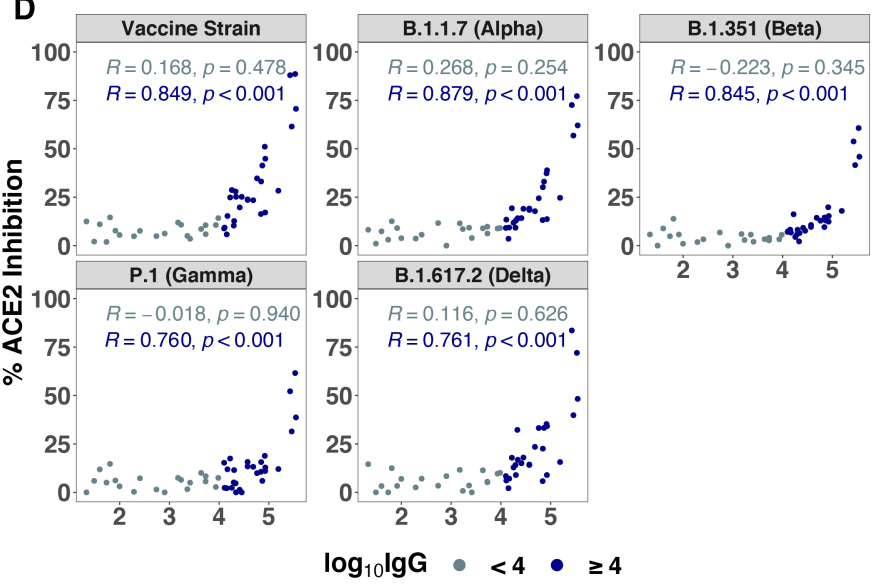



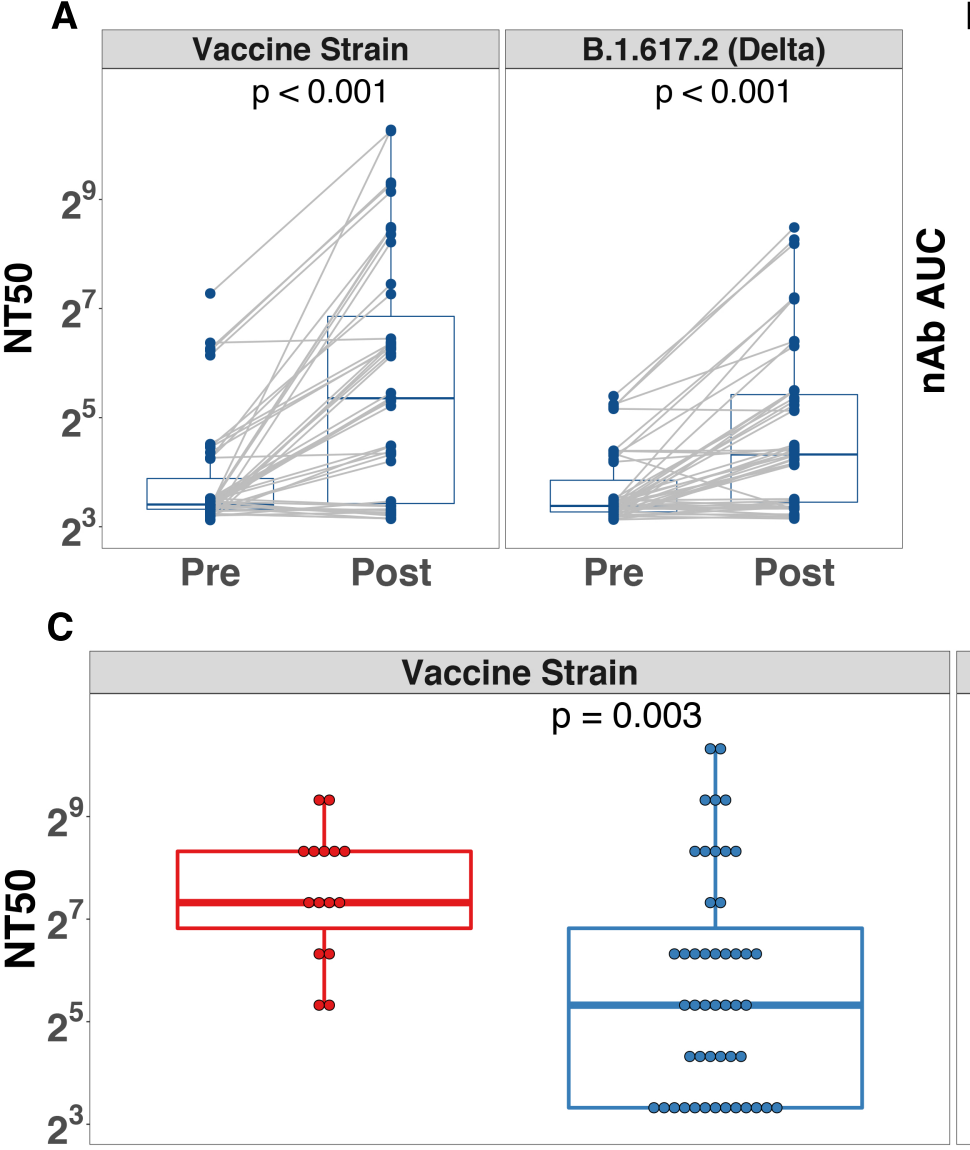

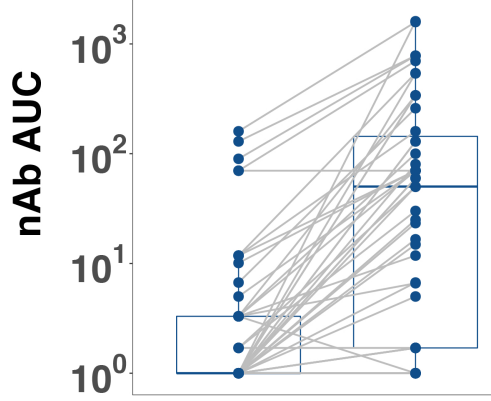

Pre
Vaccine Strain $p<0.001$

Post
B.1.617.2 (Delta) $p<0.001$

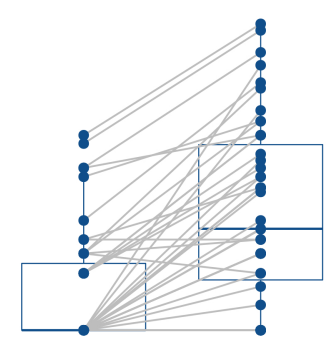

Pre Post
B.1.617.2 (Delta) $p<0.001$
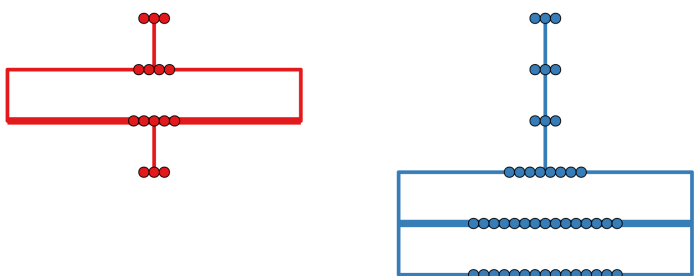

Cohort - HCs • SOTRs

D Vaccine Strain $p=0.001$ $\underbrace{10^{3}}_{10^{1}}$
$\underbrace{}_{10^{\circ}}$
$\underbrace{2}$

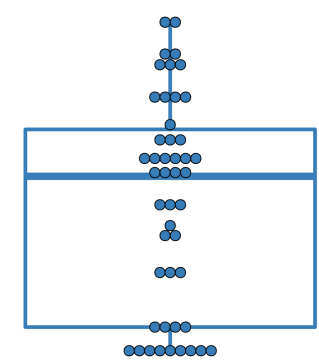

B.1.617.2 (Delta) $p<0.001$
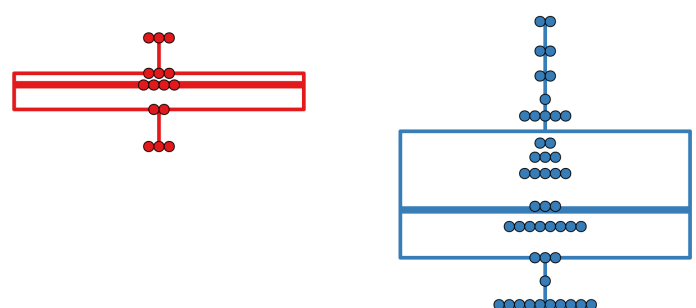


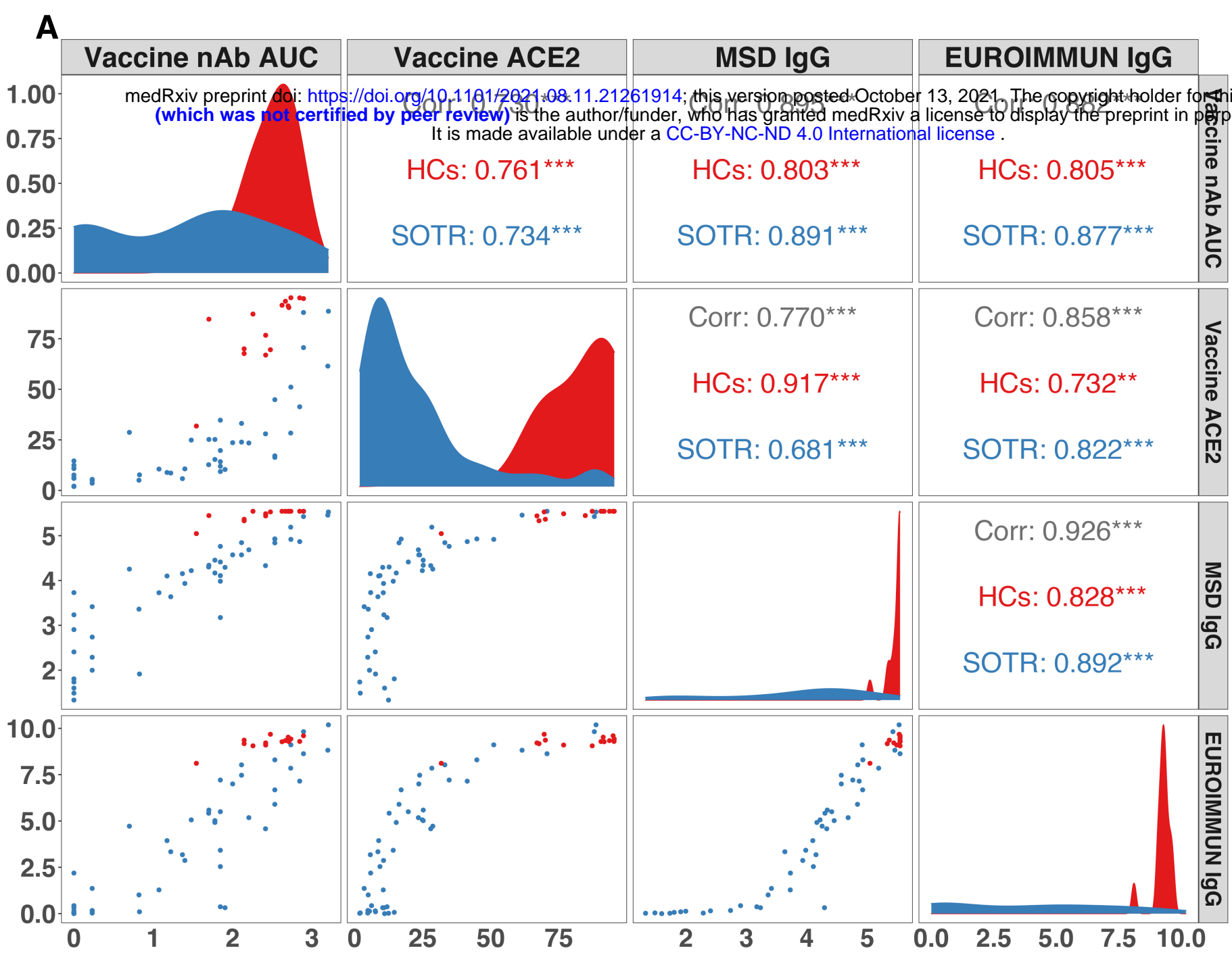

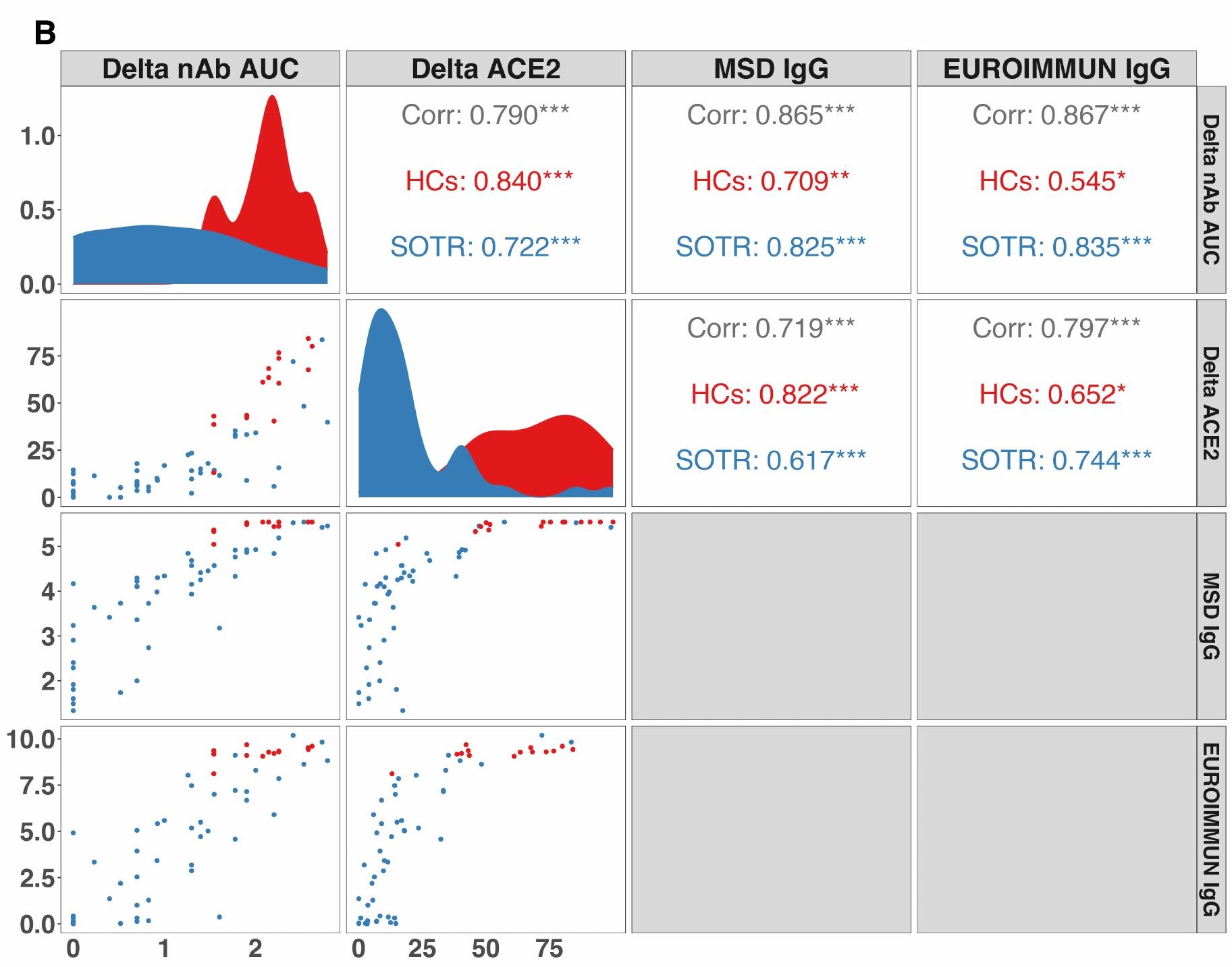

